\title{
On the Issue of Reinstatement in Argumentation
}

Martin Caminada

Department of Information and Computing Sciences, Utrecht University Technical Report UU-CS-2006-023

www.cs.uu.nl

ISSN: 0924-3275 


\title{
On the Issue of Reinstatement in Argumentation
}

\author{
Martin W.A. Caminada
}

May 1, 2006

\begin{abstract}
Dung's theory of abstract argumentation frameworks [8] led to the formalization of various argument-based semantics, which are actually particular forms of dealing with the issue of reinstatement. In this paper, we re-examine the issue of semantics from the perspective of postulates. In particular, we ask ourselves the question of which (minimal) requirements have to be fulfilled by any principle for handling reinstatement, and how this relates to Dung's standard semantics. Our purpose is to shed new light on the ongoing discussion on which semantics is most appropriate.
\end{abstract}

\section{Introduction}

Dung's abstract theory of formal argumentation [8] has been a guide for researchers in the field of formal argumentation and nonmonotonic logic for more than ten years. During this period, a significant amount of work has been done on proof procedures for Dung's various argument-based semantics $[19,5]$, as well as on concrete argumentation formalisms (such as $[15,9,1]$ based on Dung's theory.

One specific issue that has received relatively little attention is the nature of reinstatement. Although reinstatement as a principle is not totally uncontroversial [11], the current consensus among many researchers in formal argumentation and nonmonotonic logic is that reinstatement of arguments is an essential feature of defeasible reasoning (as is for instance expressed in [13]). Dung provides several approaches for dealing with reinstatement, like stable semantics, preferred semantics, complete semantics and grounded semantics. Our contribution is not to criticize Dung's theory but rather to strengthen it. In particular, we ask ourselves the question: "Why do these semantics actually make sense?"

In previous work, we have stated a number of postulates which, in our view, every argumentation formalism should satisfy [4]. In the current paper, we will follow the same approach and state some simple and intuitive properties for dealing with the issue of reinstatement We then show how these properties are satisfied by Dung's standard semantics and how the differences between the various semantics could be viewed. We also show that a careful examination of reinstatement postulates reveals a semantics not currently known. Based on this discussion, we then share some thoughts on which type of semantics is most appropriate.

\section{Dung's Standard Semantics}

A central notion in Dung's theory of abstract argumentation [8] is that of an argumentation framework, which is defined as follows. 
Definition 1 (argumentation framework). An argumentation framework is a pair (Ar, def) where $A r$ is a set of arguments and def $\subseteq A r \times A r$.

The shorthand notation $A^{+}$and $A^{-}$stands for, respectively, the set of arguments defeated by $A$ and the set of arguments that defeat $A$.

Definition 2 (defense / conflict-free). Let $A \in A r$ and $\mathcal{A} r g s \subseteq A r$.

- We define $A^{+}$as $\{B \mid A$ def $B\}$ and $\mathcal{A r g s}^{+}$as $\{B \mid A$ def $B$ for some $A \in \mathcal{A}$ rgs $\}$.

- We define $A^{-}$as $\{B \mid B \operatorname{def} A\}$ and Args $^{-}$as $\{B \mid B$ def $A$ for some $A \in \mathcal{A}$ rgs $\}$.

- Args defends an argument $A$ iff $A^{-} \subseteq \mathcal{A r g s}^{+}$.

- $\mathcal{A}$ rgs is conflict-free iff $\mathcal{A}$ rgs $\cap \mathcal{A r g s}^{+}=\emptyset$.

In the following definition, $F(\mathcal{A}$ rgs $)$ stands for the set of arguments that are acceptable (in the sense of [8]) with respect to $\mathcal{A}$ rgs.

Definition 3 (acceptability semantics). Let $\mathcal{A}$ rgs be a conflict-free set of arguments and let $F: 2^{\text {Args }} \rightarrow 2^{\text {Args }}$ be a function such that $F(\mathcal{A}$ rgs $)=\{A \mid A$ is defended by Args $\}$.

- $\mathcal{A}$ rgs is admissible iff $\mathcal{A} r g s \subseteq F(\mathcal{A} r g s)$.

- Args is a complete extension iff $\mathcal{A}$ rgs $=F($ Args $)$.

- Args is a grounded extension iff Args is the minimal (w.r.t. set-inclusion) complete extension.

- Args is a preferred extension iff Args is a maximal (w.r.t. set-inclusion) complete extension.

- Args is a stable extension iff Args is a preferred extension that defeats every argument in Ar $\backslash$ Args.

Note that there is only one grounded extension. It contains all the arguments which are not defeated, as well as those arguments which are directly or indirectly defended by non-defeated arguments.

\section{Reinstatement Labellings}

The issue of quality postulates, or axioms, has recently received some attention in the field of formal argumentation and non-monotonic logic $[4,3]$. An interesting question is whether one can also provide quality postulates for dealing with the reinstatement of arguments. Although the reinstatement has to a great extent been studied by Dung [8], the issue of which postulates have to be satisfied in order for a specific criterion for reinstatement to make sense has received relatively little attention.

One possible approach would be to start labelling the arguments in an argumentation framework. We distinguish three labels: "in", "out" and "undec" (undecided). 
Definition 4. Let (Ar, def) be a Dung-style argumentation framework. An AF-labelling is a (total) function $\mathcal{L}: A r \longrightarrow\{$ in, out, undec $\}$. We define $\operatorname{in}(\mathcal{L})$ as $\{A \in A r \mid \mathcal{L}(A)=$ in $\}$, $\operatorname{out}(\mathcal{L})$ as $\{A \in \operatorname{Ar} \mid \mathcal{L}(A)=$ out $\}$ and $\operatorname{undec}(\mathcal{L})$ as $\{A \in A r \mid \mathcal{L}(A)=$ undec $\}$.

In a reinstatement labelling, an argument is "in" iff all its defeaters are "out" and an argument is "out" if it has a defeater that is "in", as is stated in the following definition.

Definition 5. Let $\mathcal{L}$ be an AF-labelling. We say that $\mathcal{L}$ is a reinstatement labelling iff it satisfies the following:

- $\forall A \in A r:(\mathcal{L}(A)=$ out $\equiv \exists B \in A r:(B \operatorname{def} A \wedge \mathcal{L}(B)=$ in $))$ and

- $\forall A \in A r:(\mathcal{L}(A)=$ in $\equiv \forall B \in A r:(B \operatorname{def} A \supset \mathcal{L}(B)=$ out $))$

The above definitions can be illustrated using the argumentation frameworks in figure 1. In the leftmost argumentation framework, there exists just one reinstatement labelling $\left(\mathcal{L}_{1}\right)$ with $\mathcal{L}_{1}(A)=$ in, $\mathcal{L}_{1}(B)=$ out, $\mathcal{L}_{1}(C)=$ in. In the middle argumentation framework, there exist three reinstatement labellings $\left(\mathcal{L}_{2}, \mathcal{L}_{3}, \mathcal{L}_{4}\right)$ with $\mathcal{L}_{2}(D)=$ in, $\mathcal{L}_{2}(E)=$ out, $\mathcal{L}_{3}(D)=$ out, $\mathcal{L}_{3}(E)=$ in, $\mathcal{L}_{4}(D)=$ undec and $\mathcal{L}_{4}(E)=$ undec. In the rightmost argumentation framework, there exists just one reinstatement labelling $\left(\mathcal{L}_{5}\right)$ with $\mathcal{L}_{5}(F)=$ undec.

Notice that definition 5 can actually be seen as a postulate, as it specifies a restriction on an AF-labelling. It turns out that different kinds of reinstatement labellings correspond with different kind of Dung-style semantics. This is explored in the remainder of this paper.

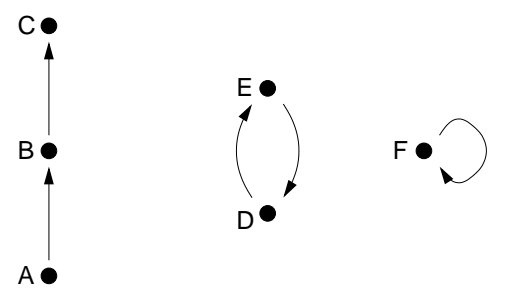

Figure 1: Three argumentation frameworks.

\section{Labellings versus Semantics}

We now define two functions that, given an argumentation framework, allow a set of arguments to be converted to a labelling and vice versa. The function Ext2Lab $(A r, d e f)$ takes a set of arguments (sometimes an extension) and converts it to a labelling. The function Lab2Ext $_{(A r, d e f)}$ takes an AF-labelling and converts it to a set of arguments (sometimes an extension). Notice that as an AF-labelling is defined as a function (definition 4), which in its turn is essentially a relation, it is possible to represent the labelling as a set of pairs.

In the following definition, the resulting AF-labelling does not yet need to satisfy the properties of a reinstatement labelling as stated in definition 5 .

Definition 6. Let $(A r$, def $)$ be an argumentation framework, Args $\subseteq$ Ar such that $\mathcal{A r g s}$ is conflict-free, and $\mathcal{L}: A r \longrightarrow\{$ in, out, undec $\}$ an $A$ F-labelling. We define $\operatorname{Ext}_{2} \mathrm{Lab}_{(A r, \text { def })}(\mathcal{A}$ rgs $)$ as $\{(A$, in $) \mid A \in \mathcal{A} \operatorname{Args}\} \cup\left\{(A\right.$, out $\left.) \mid \exists A^{\prime} \in \mathcal{A} \operatorname{Args}: A^{\prime} \operatorname{def} A\right\} \cup\left\{(A\right.$, undec $) \mid A \notin \mathcal{A}$ rgs $\wedge \neg \exists A^{\prime} \in$ Args : $\left.A^{\prime} \operatorname{def} A\right\}$. We define LabToExt Ler,def $)_{(\mathcal{L})}$ as $\{A \mid(A$, in $) \in \mathcal{L}\}$. 
The fact that $\mathcal{A}$ rgs is conflict-free in the above definition makes that $\operatorname{Ext2Lab}_{(A r, d e f)}(\mathcal{A r g s})$ is indeed an AF-labelling. When the associated argumentation framework is clear, we some-

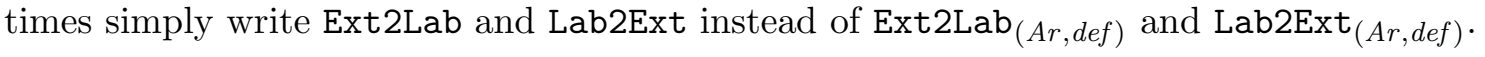

\subsection{Reinstatement labellings without restrictions}

It is interesting to notice that a reinstatement labelling coincides with Dung's notion of a complete extension. This is stated by the theorems 1 and 2 .

Theorem 1. Let $(A r$, def $)$ be an argumentation framework and let $\mathcal{L}$ be a reinstatement labelling. Then Lab2Ext $(\mathcal{L})$ is a complete extension.

Proof. Let $\mathcal{A}$ rgs $=\operatorname{Lab2\operatorname {Ext}}(\mathcal{L})$. We now prove that $\mathcal{A}$ rgs is a complete extension, that is, $F(\mathcal{A}$ rgs $)=\mathcal{A}$ rgs. For this, we prove two things.

1. $\mathcal{A r g s} \subseteq F($ Args $)$

Let $A \in \mathcal{A}$ rgs. Then $\mathcal{L}(A)=$ in. The fact that $\mathcal{L}$ is a reinstatement labelling means that each defeater $B$ of $A$ is labeled out. This again means (still by the fact that $\mathcal{L}$ is a reinstatement labelling) that each such $B$ has a defeater (say $C$ ) that is labeled in. By definition of Lab2Ext, it holds that $C \in \mathcal{A}$ rgs. This means that for each defeater $B$ of $A$, there is a $C \in \mathcal{A}$ rgs that defeats $B$. Therefore, $A \in F(\mathcal{A r g s})$ ( $A$ is defended by Args).

2. $F($ Args $) \subseteq \mathcal{A}$ rgs

Let $A \in F(\mathcal{A}$ rgs $)$. Then each $B$ that defeats $A$ is defeated by some $C \in \mathcal{A}$ rgs means by definition of Lab2Ext that $C$ is labeled in by $\mathcal{L}$. The fact that $\mathcal{L}$ is a reinstatement labelling means that $B$ is labeled out. This again means that $A$ is labeled in. Therefore, by definition of Lab2Ext, $A \in \mathcal{A}$ rgs .

Theorem 2. Let $(A r$, def $)$ be an argumentation framework and let Args be a complete extension. Then $\mathcal{L}=\operatorname{Ext} 2 \mathrm{Lab}(\mathcal{A}$ rgs $)$ is a reinstatement labelling.

Proof. In order to prove that $\operatorname{Ext} 2 \mathrm{Lab}(\mathcal{A r g s})$ is a reinstatement labelling, we have to prove four things:

1. $\forall A \in A r:(\mathcal{L}(A)=$ out $\supset \exists B \in A r:(B \operatorname{def} A \wedge \mathcal{L}(B)=$ in $))$

Let $A \in A r$ such that $\mathcal{L}(A)=$ out. Then, according to the definition of Ext2Lab, the fact that $\mathcal{L}(A)=$ out means that there is an $A^{\prime} \in \mathcal{A}$ rgs that defeats $A$. And the fact that $A^{\prime} \in \mathcal{A}$ rrgs means that $\mathcal{L}\left(A^{\prime}\right)=$ in.

2. $\forall A \in A r:(\mathcal{L}(A)=$ out $\subset \exists B \in A r:(B \operatorname{def} A \wedge \mathcal{L}(B)=$ in $))$

Let $A \in A r$ be such that it has a defeater $B$ labeled in. The fact that $\mathcal{L}(B)=$ in means that $B \in \mathcal{A}$ rgs (Ext2Lab). By definition of Ext2Lab, $\mathcal{L}(A)=$ out.

3. $\forall A \in A r:(\mathcal{L}(A)=$ in $\supset \forall B \in A r:(B \operatorname{def} A \supset \mathcal{L}(B)=$ out $))$

Let $A$ be an argument that is labeled in. The fact that $\mathcal{L}(A)=$ in means that $A \in \mathcal{A}$ rgs. The fact that $\mathcal{A}$ rgs is a complete extension implies that it is an admissible set. That is, $\mathcal{A}$ rgs defeats every defeater of $A$. By the definition of Ext2Lab, this means that every defeater of $A$ is labeled out. 
4. $\forall A \in A r:(\mathcal{L}(A)=$ in $\subset \forall B \in A r:(B \operatorname{def} A \supset \mathcal{L}(B)=$ out $))$

Let $A \in A r$ be such that every defeater of $A$ is labeled out. This means, by definition of Ext2Lab, that for every defeater $B$ of $A$ there is a $C \in \mathcal{A}$ rgs that defeats $B$. But as $\mathcal{A}$ rgs is a complete extension (everything that is defended by $\mathcal{A}$ rgs is already in $\mathcal{A}$ rgs) this means that $A \in \mathcal{A}$ rgs. By definition of Ext2Lab, this means that $A$ is labeled in.

It is interesting to observe that, when the domain and range of Lab2Ext is restricted to reinstatement labellings and complete extensions, and the domain and range of Ext2Lab is restricted to complete extensions and reinstatement labellings, then the resulting functions (call them Lab2Ext ${ }^{r}$ and Ext2Lab ${ }^{r}$ ) are bijective (that is, they are both injective and surjective) and each other's inverse.

\section{Theorem 3.}

Let Lab2Ext $_{(\text {Ar,def })}^{r}:\{\mathcal{L} \mid \mathcal{L}$ is a reinstatement labelling of $($ Ar, def $)\} \longrightarrow\{$ Args $\mid$ Args is a complete extension of $(A r$, def $)\}$ be a function defined by Lab2Ext ${ }_{(A r, \text { def })}(\mathcal{L})=$ $\operatorname{Lab2Ext}_{(A r, d e f)}(\mathcal{L})$.

Let $\operatorname{Ext2Lab}_{(A r, \text { def })}^{r}:\{\mathcal{A}$ rgs $\mid \mathcal{A}$ rgs is a complete extension of $($ Ar, def $)\} \longrightarrow\{\mathcal{L} \mid \mathcal{L}$ is a reinstatement labelling of $(A r$, def $)\}$ be a function defined by $\operatorname{Ext}_{2 \operatorname{Lab}_{(A r, d e f}^{r}}^{r}(\mathcal{A r g s})=$ $\operatorname{Ext2Lab}_{(A r, d e f)}($ Args $)$.

The functions Lab2Ext ${ }^{r}$ and $\mathrm{Ext}_{2} \mathrm{Lab}^{r}$ are bijective and are each other's inverse.

Proof. As every function that has an inverse is bijective, we only need to prove that Lab2Ext ${ }^{r}$ and Ext2Lab ${ }^{r}$ are each other's inverses. That is $\left(\mathrm{Lab}_{2} \mathrm{Ext}^{r}\right)^{-1}=\mathrm{Ext}_{2} \mathrm{Lab}^{r}$ and $\left(\mathrm{Ext}_{2 \mathrm{Lab}}{ }^{r}\right)^{-1}=\mathrm{Lab}^{\mathrm{Ext}}{ }^{r}$. For this, we prove the following two things:

1. For every reinstatement labelling $\mathcal{L}$ it holds that

$\operatorname{Ext} 2 \operatorname{Lab}^{r}\left(\operatorname{Lab} 2 \operatorname{Ext}^{r}(\mathcal{L})\right)=\mathcal{L}$.

Let $\mathcal{L}$ be a reinstatement labelling of $(A r, \operatorname{def})$ and let $A \in A r$.

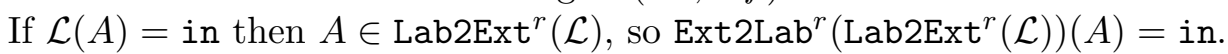

If $\mathcal{L}(A)=$ out then $A$ is defeated by $\operatorname{Lab} \operatorname{Ext}^{r}(\mathcal{L})$, so $\operatorname{Ext2Lab}^{r}\left(\operatorname{Lab}^{2} \operatorname{Ext}^{r}(\mathcal{L})\right)(A)=$ out.

If $\mathcal{L}(A)=$ undec then $A \notin \operatorname{Lab}^{2} \operatorname{Ext}^{r}(\mathcal{L})$ and $A$ is not defeated by Lab2Ext ${ }^{r}(\mathcal{L})$, so

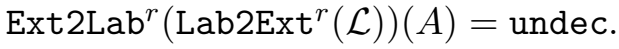

2. For every complete extension $\mathcal{A}$ rgs it holds that

Lab2Ext ${ }^{r}\left(\operatorname{Ext}_{2 L^{2}}{ }^{r}(\mathcal{A} r g s)\right)=\mathcal{A}$ rgs .

Let $\mathcal{A} r g s$ be a complete extension of $(A r, d e f)$. We now prove two things:

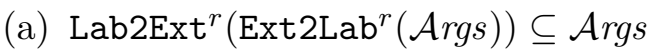

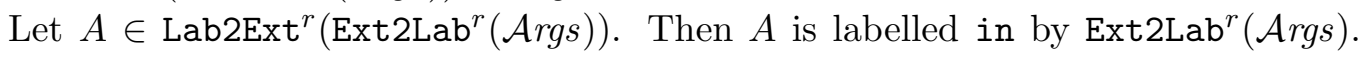
Therefore $A \in$ Args.

(b) $\mathcal{A} r g s \in \operatorname{Lab} \operatorname{Ext}^{r}\left(\operatorname{Ext}_{2} \operatorname{Lab}^{r}(\mathcal{A}\right.$ rgs $\left.)\right) \subseteq \mathcal{A}$ Args

Let $A \in \mathcal{A}$ rgs. Then $A$ is labelled in by Ext2Lab ${ }^{r}(\mathcal{A}$ rgs $)$. Therefore $A \in$

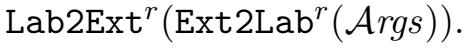

As Lab2Ext ${ }^{r}$ and Ext2Lab ${ }^{r}$ are bijective functions that are each other's inverse, there exists a strong similarity between complete extensions and reinstatement labellings. 


\subsection{Reinstatement labellings with empty undec}

Reinstatement labellings where undec is empty coincide with stable extensions. This is stated by the theorems 4 and 5 .

Theorem 4. Let $(A r$, def $)$ be an argumentation framework and let $\mathcal{L}$ be a reinstatement labelling such that undec $(\mathcal{L})=\emptyset$. Then $\operatorname{Lab2\operatorname {Ext}}(\mathcal{L})$ is a stable extension.

Proof. Let $\mathcal{A} r g s=\operatorname{Lab2\operatorname {Ext}}(\mathcal{L})$. Now consider an arbitrary $A \in A r \backslash \mathcal{A}$ rgs. From the fact that undec $(\mathcal{L})=\emptyset$, it follows that $\mathcal{L}(A)=$ out. By definition, this means that $A$ is defeated by an argument (say $B$ ) labelled in. The fact that $B$ is labelled in means that $B \in \mathcal{A}$ rgs. Therefore, $A$ is defeated by some argument in $\mathcal{A}$ rgs. As this holds for any arbitrary $A \in A r \backslash \mathcal{A} r g s$, it means that $\mathcal{A}$ rgs defeats any argument not in it. Thus, $\mathcal{A}$ rgs is a stable extension.

Theorem 5. Let (Ar, def) be an argumentation framework and let $\mathcal{A}$ rgs be a stable extension. Then $\mathcal{L}=\operatorname{Ext} 2 \operatorname{Lab}(\mathcal{A}$ rgs $)$ is a labelling such that undec $(\mathcal{L})=\emptyset$.

Proof. Let $A \in A r$. We distinguish two possibilities:

1. $A \in \mathcal{A}$ rgs. Then, by definition, $\mathcal{L}(A)=$ in.

2. $A \notin \mathcal{A}$ rgs. As $\mathcal{A}$ rgs is a stable extension, this means that some argument in $\mathcal{A}$ rgs defeats $A$. This means that $\mathcal{L}(A)=$ out.

In both cases, $\mathcal{L}(A) \neq$ undec. As this holds for any arbitrary $A \in A r$, it holds that undec $(\mathcal{L})=$ $\emptyset$.

\subsection{Reinstatement labellings with maximal in}

Reinstatement labellings where in is maximal coincide with preferred extensions. This is stated by the theorems 6 and 7 .

Theorem 6. Let $(A r$, def $)$ be an argumentation framework and let $\mathcal{L}$ be a reinstatement labelling such that $\operatorname{in}(\mathcal{L})$ is maximal. Then Lab2Ext $(\mathcal{L})$ is a preferred extension.

Proof. Let $\mathcal{L}$ be a reinstatement labelling such that $\operatorname{in}(\mathcal{L})$ is maximal. Now, suppose that $\mathcal{A} r g s=\operatorname{Lab2Ext}(\mathcal{L})$ is not a preferred extension. Then, by definition of a preferred extension (definition 3) there must be a complete extension $\mathcal{A r g s}{ }^{\prime}$ such that $\mathcal{A r g s} \subsetneq \mathcal{A} \mathcal{A r g s}^{\prime}$. Let $\mathcal{L}^{\prime}=\operatorname{Ext} 2 \operatorname{Lab}\left(\mathcal{A r g s} s^{\prime}\right)$. Then, $\operatorname{in}(\mathcal{L}) \subsetneq \operatorname{in}\left(\mathcal{L}^{\prime}\right)$. But then in $(\mathcal{L})$ would not be maximal. Contradiction.

Theorem 7. Let $(A r$, def $)$ be an argumentation framework and let $\mathcal{A}$ rgs be a preferred extension. Then $\mathcal{L}=\operatorname{Ext} 2 \operatorname{Lab}(\mathcal{A}$ rgs $)$ is a labelling such that $\operatorname{in}(\mathcal{L})$ is maximal.

Proof. Let $\mathcal{A}$ rgs be a preferred extension and let $\mathcal{L}$ be Ext2Lab $(\mathcal{A}$ rgs $)$. Now, suppose that $\operatorname{in}(\mathcal{L})$ is not maximal. Then there must be some reinstatement labelling $\mathcal{L}^{\prime}$ with $\operatorname{in}(\mathcal{L}) \subsetneq$ in $\left(\mathcal{L}^{\prime}\right)$. Let $\mathcal{A} r g s^{\prime}=\operatorname{Lab2Ext}\left(\mathcal{L}^{\prime}\right)$. Then $\mathcal{A}$ rgs $s^{\prime}$ is a complete extension with $\mathcal{A} r g s \subsetneq \mathcal{A r g s}{ }^{\prime}$. But then $\mathcal{A}$ rgs would not be a preferred extension. Contradiction. 


\subsection{Reinstatement labellings with maximal out}

It is interesting to notice that, contrary to what one might expect, reinstatement labellings in which out is maximized coincide with preferred extensions, just like (as was proved earlier) labellings in which in is maximized. We start our proofs with two lemmas.

Lemma 1. Let $\mathcal{L}$ and $\mathcal{L}^{\prime}$ be two reinstatement labellings. If $\operatorname{in}(\mathcal{L}) \subsetneq \operatorname{in}\left(\mathcal{L}^{\prime}\right)$ then out $(\mathcal{L}) \subsetneq$ $\operatorname{out}\left(\mathcal{L}^{\prime}\right)$.

Proof. Suppose in $(\mathcal{L}) \subsetneq \operatorname{in}\left(\mathcal{L}^{\prime}\right)$. This means two things:

1. $\forall A \in \operatorname{in}(\mathcal{L}): A \in \operatorname{in}\left(\mathcal{L}^{\prime}\right)$

2. $\exists B \in \operatorname{in}\left(\mathcal{L}^{\prime}\right): B \notin \operatorname{in}(\mathcal{L})$

We now prove the following two things:

- $\forall C \in \operatorname{out}(\mathcal{L}): C \in \operatorname{out}\left(\mathrm{L}^{\prime}\right)$.

Let $C \in \operatorname{out}(\mathcal{L})$. By the definition of a reinstatement labelling (definition 5 ) this means that $C$ is defeated by some $A \in \operatorname{in}(\mathcal{L})$. But then, according to 1 , it also holds that $A \in \operatorname{in}\left(\mathcal{L}^{\prime}\right)$. This, by the definition of a reinstatement labelling, means that $C \in \operatorname{out}\left(\mathcal{L}^{\prime}\right)$.

- $\exists D \in \operatorname{out}\left(\mathcal{L}^{\prime}\right): D \notin \operatorname{out}(\mathcal{L})$.

Let $B$ be an argument (taken from 2) such that $B \in \operatorname{in}\left(\mathcal{L}^{\prime}\right)$. Then, according to the definition of a reinstatement labelling, it must also be the case that each defeater of $B$ is labelled out in $\mathcal{L}^{\prime}$, but there is some defeater of $B$ that is not labelled out in $\mathcal{L}$. This means that $\exists D \in \operatorname{out}\left(\mathcal{L}^{\prime}\right): D \notin \operatorname{out}(\mathcal{L})$

Lemma 2. Let $\mathcal{L}$ and $\mathcal{L}^{\prime}$ be two reinstatement labellings. If out $(\mathcal{L}) \subsetneq \operatorname{out}\left(\mathcal{L}^{\prime}\right)$ then $\operatorname{in}(\mathcal{L}) \subsetneq$ $\operatorname{in}\left(\mathcal{L}^{\prime}\right)$.

Proof. Suppose out $(\mathcal{L}) \subsetneq \operatorname{out}\left(\mathcal{L}^{\prime}\right)$. This means two things:

1. $\forall A \in \operatorname{out}(\mathcal{L}): A \in \operatorname{out}\left(\mathcal{L}^{\prime}\right)$

2. $\exists B \in \operatorname{out}\left(\mathcal{L}^{\prime}\right): B \notin \operatorname{out}(\mathcal{L})$

We now prove the following two things:

- $\forall C \in \operatorname{in}(\mathcal{L}): C \in \operatorname{in}\left(\mathcal{L}^{\prime}\right)$

Let $C \in \operatorname{in}(\mathcal{L})$. By the definition of a reinstatement labelling (definition 5) this means that every defeater of $C$ is labelled out in $\mathcal{L}$. But then (according to 1 ) every defeater of $C$ is also labelled out in $\mathcal{L}^{\prime}$. This, by the definition of a reinstatement labelling, means that $C$ is labelled in in $\mathcal{L}^{\prime}$.

- $\exists D \in \operatorname{in}\left(\mathcal{L}^{\prime}\right): D \notin \operatorname{in}(\mathrm{L})$

Let $B$ be an argument (taken from 2 ) such that $B \in \operatorname{out}\left(\mathcal{L}^{\prime}\right)$ and $B \notin \operatorname{out}(\mathcal{L})$. Then, according to the definition of a reinstatement labelling, this means that some defeater of $B$ is labelled in in $\mathcal{L}^{\prime}$, but no defeater of $B$ is labelled in in $\mathcal{L}$. This means that $\exists D \in \operatorname{in}\left(\mathcal{L}^{\prime}\right): D \notin \operatorname{in}(\mathcal{L})$. 
Using these two lemmas, we can now state and prove the main theorems.

Theorem 8. Let $(A r$, def $)$ be an argumentation framework and let $\mathcal{L}$ be a reinstatement labelling such that out $(\mathcal{L})$ is maximal. Then $\operatorname{Lab} 2 \operatorname{Ext}(\mathcal{L})$ is a preferred extension.

Proof. Let $\mathcal{L}$ be a reinstatement labelling such that out $(\mathcal{L})$ is maximal. Now, suppose that $\operatorname{Lab2\operatorname {Ext}}(\mathcal{L})$ is not a preferred extension. Then, by theorem 6 , $\operatorname{in}(\mathcal{L})$ is not maximal. This means that there exists some $\mathcal{L}^{\prime}$ such that $\operatorname{in}(\mathcal{L}) \subsetneq \operatorname{in}\left(\mathcal{L}^{\prime}\right)$. By lemma 1 this also means that $\operatorname{out}(\mathcal{L}) \subsetneq \operatorname{out}\left(\mathcal{L}^{\prime}\right)$. But then out $(\mathcal{L})$ would not be maximal. Contradiction.

Theorem 9. Let $(A r$, def $)$ be an argumentation framework and let $\mathcal{A}$ rgs be a preferred extension. Then $\mathcal{L}=\operatorname{Ext} 2 \operatorname{Lab}(\mathcal{A} r g s)$ is a labelling such that out $(\mathcal{L})$ is maximal.

Proof. Let $\mathcal{A}$ rgs be a preferred extension. Then, by theorem 7, $\operatorname{Ext2Lab}(\mathcal{A} r g s)$ is a labelling $(\mathcal{L})$ such that $\operatorname{in}(\mathcal{L})$ is maximal. Now suppose that out $(\mathcal{L})$ is not maximal. Then there exists some reinstatement labelling $\mathcal{L}^{\prime}$ with $\operatorname{out}(\mathcal{L}) \subsetneq \operatorname{out}\left(\mathcal{L}^{\prime}\right)$ By lemma 2 , this also means that $\operatorname{in}(\mathcal{L}) \subseteq$ in $\left(\mathcal{L}^{\prime}\right)$. But then in $(\mathcal{L})$ would not be maximal. Contradiction.

\subsection{Reinstatement labellings with maximal undec}

A reinstatement labelling where undec is maximal coincides with the grounded extension. This is stated by the theorems 10 and 11 .

Theorem 10. Let $(A r$, def $)$ be an argumentation framework and $\mathcal{L}$ be a reinstatement labelling such that undec $(\mathcal{L})$ is maximal. Then $\operatorname{Lab2\operatorname {Ext}}(\mathcal{L})$ is the grounded extension.

Proof. (by contraposition) Suppose Lab2Ext $(\mathcal{L})$ is not the grounded extension $(G E)$. Then it must be a strict superset of the grounded extension (which, by definition, is the smallest complete extension). That is: $G E \subsetneq \operatorname{Lab2\operatorname {Ext}}(\mathcal{L})$. Let $\mathcal{L}^{\prime}=\operatorname{Ext} 2 \operatorname{Lab}(G E)$. From $G E \subsetneq$ $\operatorname{Lab2\operatorname {Ext}}(\mathcal{L})$ it follows directly that $\operatorname{in}\left(\mathcal{L}^{\prime}\right) \subsetneq \operatorname{in}(\mathcal{L})$. From lemma 1 it follows that out $\left(\mathcal{L}^{\prime}\right) \subsetneq$ $\operatorname{out}(\mathcal{L})$. Therefore, it holds that undec $(\mathcal{L}) \subsetneq \operatorname{undec}\left(\mathcal{L}^{\prime}\right)$. But then undec $(\mathcal{L})$ would not be maximal.

Theorem 11. Let $(A r$, def) be an argumentation framework and $\mathcal{A}$ rgs be the grounded extension in this framework. Then $\operatorname{Ext} 2 \mathrm{Lab}(\mathcal{A}$ rgs $)$ is a reinstatement labelling where undec $(\mathcal{L})$ is maximal.

Proof. Let $\mathcal{A}$ rgs ${ }^{\prime}(\neq \mathcal{A}$ rgs $)$ be an arbitrary complete extension. As the grounded extension is the smallest complete extension, it follows that $\mathcal{A}$ rgs $\subsetneq \mathcal{A}$ rgs ${ }^{\prime}$. Let $\mathcal{L}=\operatorname{Ext} 2 \operatorname{Lab}(\mathcal{A} r g s)$ and $\mathcal{L}^{\prime}=\operatorname{Ext} 2 \operatorname{Lab}\left(\mathcal{A} r g s^{\prime}\right)$. From $\mathcal{A} r g s \subsetneq \mathcal{A} r g s^{\prime}$ it directly follows that $\operatorname{in}(\mathcal{L}) \subsetneq \operatorname{in}\left(\mathcal{L}^{\prime}\right)$. This also means (by lemma 1) that out $(\mathcal{L}) \subsetneq \operatorname{out}\left(\mathcal{L}^{\prime}\right)$. Therefore, it holds that undec $\left(\mathcal{L}^{\prime}\right) \subsetneq$ undec $(\mathcal{L})$. As this result holds for arbitrary $\mathcal{A} r g s^{\prime}(\neq \mathcal{A}$ rgs $)$ (and therefore also for arbitrary $\mathcal{L}^{\prime}(\neq \mathcal{L})$ ), it holds that undec $(\mathcal{L})$ is maximal. 


\subsection{Reinstatement labellings with minimal in}

A reinstatement labelling with minimal in coincides with the grounded extension. This is stated by the theorems 12 and 13 .

Theorem 12. Let $(A r$, def $)$ be an argumentation framework and $\mathcal{L}$ be a reinstatement labelling such that $\operatorname{in}(\mathcal{L})$ is minimal. Then $\operatorname{Lab} 2 \operatorname{Ext}(\mathcal{L})$ is the grounded extension.

Proof. Let $\mathcal{L}$ be a reinstatement labelling such that in $(\mathcal{L})$ is minimal. Now, suppose $\mathcal{A}$ rgs $=$ Lab2Ext $(\mathcal{L})$ is not the grounded extension. Then, according to the definition of the grounded extension (definition 3) there must be some complete extension $\mathcal{A r g s}{ }^{\prime}$ with $\mathcal{A r g s}^{\prime}$ with $\mathcal{A r g s}^{\prime} \subsetneq$ Args. Let $\mathcal{L}^{\prime}=\operatorname{Ext} 2 \operatorname{Lab}\left(\mathcal{A r g s} s^{\prime}\right)$. Then $\operatorname{in}\left(\mathcal{L}^{\prime}\right) \subsetneq \operatorname{in}(\mathcal{L})$. But then $\operatorname{in}(\mathcal{L})$ would not be minimal. Contradiction.

Theorem 13. Let $(A r$, def) be an argumentation framework and $\mathcal{A}$ rgs be the grounded extension in this framework. Then $\operatorname{Ext} 2 \mathrm{Lab}(\mathcal{A}$ rgs $)$ is a reinstatement labelling where $\operatorname{in}(\mathcal{L})$ is minimal.

Proof. Let $\mathcal{A}$ rgs be the grounded extension. Let $\mathcal{L}=\operatorname{Ext} 2 \operatorname{Lab}(\mathcal{A} r g s)$. Now, suppose in $(\mathcal{L})$ is not minimal. Then there exists some $\mathcal{L}^{\prime}$ with $\operatorname{in}\left(\mathcal{L}^{\prime}\right) \subsetneq \operatorname{in}(\mathcal{L})$. Now, let $\mathcal{A r g s}{ }^{\prime}=\operatorname{Lab2Ext}\left(\mathcal{L}^{\prime}\right)$. It now holds that $\mathcal{A}$ rgs $\subsetneq \mathcal{A}$ rgs. But then $\mathcal{A}$ rgs would not be a grounded extension. Contradiction.

\subsection{Reinstatement labellings with minimal out}

A reinstatement labelling with minimal out coincides with the grounded extension. This is stated by the theorems 14 and 15 .

Theorem 14. Let $(A r$, def $)$ be an argumentation framework and $\mathcal{L}$ be a reinstatement labelling such that out $(\mathcal{L})$ is minimal. Then Lab2Ext $(\mathcal{L})$ is the grounded extension.

Proof. Let $\mathcal{L}$ be a reinstatement labelling such that out $(\mathcal{L})$ is minimal. Then, according to lemma $1 \operatorname{in}(\mathcal{L})$ is also minimal. Then, by theorem 12 , Lab2Ext $(\mathcal{L})$ is the grounded extension.

Theorem 15. Let $(A r$, def $)$ be an argumentation framework and $\mathcal{A}$ rgs be the grounded extension in this framework. Then $\operatorname{Ext} 2 \mathrm{Lab}(\mathcal{A} r g s)$ is a reinstatement labelling where out $(\mathcal{L})$ is minimal.

Proof. Let $\mathcal{A}$ rgs be the grounded extension. Let $\mathcal{L}=\operatorname{Ext} 2 \operatorname{Lab}(\mathcal{L})$. Then, by theorem 13, $\operatorname{in}(\mathcal{L})$ is minimal. Then, by lemma 2 , out $(\mathcal{L})$ is also minimal.

\subsection{Reinstatement labellings with minimal undec}

The last remaining case to be examined is the one of reinstatement labellings where undec is minimized. We show that this does not coincide with any of Dung's standard semantics.

There is a one-way relation between reinstatement labellings with minimal undec and preferred extensions, as is stated in the following theorem.

Theorem 16. Let $(A r$, def $)$ be an argumentation framework and $\mathcal{L}$ be a reinstatement labelling such that undec $(\mathcal{L})$ is minimal. Then Lab2Ext $(\mathcal{L})$ is a preferred extension. 
Proof. (reductio ad absurdum) Suppose $\operatorname{Args}=\operatorname{Lab2\operatorname {Ext}}(\mathcal{L})$ is not a preferred extension. Then there exists an admissible set $\mathcal{A} \operatorname{Args}^{\prime}$ that is a strict superset of $\mathcal{A} \operatorname{Arg}\left(\mathcal{A} \operatorname{Args} \subsetneq \mathcal{A} \operatorname{Args}^{\prime}\right)$. Now consider $\operatorname{Ext} 2 \operatorname{Lab}\left(\mathcal{A r g s} s^{\prime}\right)=\mathcal{L}^{\prime}$. Obviously, it holds that $\operatorname{in}(\mathcal{L}) \subsetneq \operatorname{in}\left(\mathcal{L}^{\prime}\right)$. This also implies (lemma 1) that out $(\mathcal{L}) \subsetneq \operatorname{out}\left(\mathcal{L}^{\prime}\right)$. From the facts that in $(\mathcal{L}) \subsetneq \operatorname{in}\left(\mathcal{L}^{\prime}\right)$ and out $(\mathcal{L}) \subsetneq \operatorname{out}\left(\mathrm{L}^{\prime}\right)$, it follows that undec $\left(\mathcal{L}^{\prime}\right) \subsetneq$ undec $(\mathcal{L})$. But then undec $(\mathcal{L})$ is not minimal. Contradiction.

Unfortunately, it doesn't work the other way around. If $\mathcal{A} r g s$ is a preferred extension, then it is not necessarily the case that $\operatorname{Ext} 2 \mathrm{Lab}(\mathcal{A}$ rgs $)$ is a reinstatement labelling where undec $(\mathcal{L})$ is minimal. This is shown in the following example.

Example 1. Let $A r=\{A, B, C, D, E\}$ and let $A$ defeat $B, B$ defeat $A, B$ defeat $C, C$ defeat $D, D$ defeat $E$, and $E$ defeat $C$ (see also figure 2). Here, there exists two preferred extensions: $\mathcal{E}_{1}=\{B, D\}$ and $\mathcal{E}_{2}=\{A\}$. As $\mathcal{E}_{1}$ is also a stable extension, it holds that $\operatorname{Ext} 2 \mathrm{Lab}\left(\mathcal{E}_{1}\right)$ yields a labelling (say $\mathcal{L})$ with undec $(\mathcal{L})=\emptyset$. However, $\operatorname{Ext} 2 \operatorname{Lab}\left(\mathcal{E}_{2}\right)$ yields a labelling (say $\left.\mathcal{L}^{\prime}\right)$ with undec $\left(\mathcal{L}^{\prime}\right)=\{C, D, E\}$. So, even though $\mathcal{E}_{2}$ is a preferred extension, $\operatorname{Ext} 2 \operatorname{Lab}\left(\mathcal{E}_{2}\right)$ is not a reinstatement labelling in which undec is minimal.

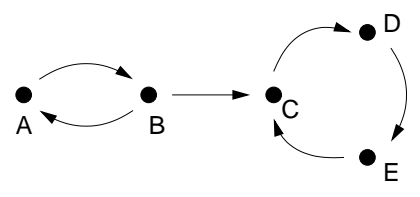

Figure 2: A preferred extension does not necessarily imply minimal undec.

Before continuing with our analysis, we first state two helpful lemmas.

Lemma 3. Let $(A r$, def $)$ be an argumentation framework and Args be a complete extension. Let $\mathcal{L}=\operatorname{Ext} 2 \mathrm{Lab}(\mathcal{A}$ rgs $)$. Then:

1. $\operatorname{in}(\mathcal{L})=\mathcal{A r g s}$

2. $\operatorname{out}(\mathcal{L})=\mathcal{A r g s}^{+}$

3. $\operatorname{undec}(\mathcal{L})=\operatorname{Ar} \backslash\left(\operatorname{Args} \cup \mathcal{A r g s}^{+}\right)$

Proof. This follows directly from the definition of Ext2Lab (definition 6).

Lemma 4. Let $(A r, d e f)$ be an argumentation framework and let $\mathcal{L}$ be a reinstatement la-

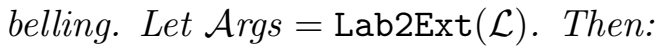

1. $\operatorname{Args}=\operatorname{in}(\mathcal{L})$

2. $\mathcal{A r g s}^{+}=\operatorname{out}(\mathcal{L})$

3. $\operatorname{Ar} \backslash\left(\mathcal{A r g s} \cup \mathcal{A r g s}^{+}\right)=\operatorname{undec}(\mathcal{L})$

Proof.

1. This follows directly from the definition of Lab2Ext (definition 6). 
2. This follows from 1 and the definition of a reinstatement labelling (definition 5).

3. This follows from 1 and 2 , together with the fact that a reinstatement labelling is a total function (it assigns exactly one label from \{in, out, undec $\}$ to each argument).

Labellings in which undec is minimized can be seen as produced by an agent that is eager to take a position (in or out) on as many arguments as possible. It is not too difficult to specify what these would look like as a Dung-style semantics.

Definition 7. Let $(A r$, def $)$ be an argumentation framework and $\mathcal{A r g s} \subseteq$ Ar. Args is called a semi-stable extension iff $\mathcal{A}$ rgs is a complete extension where $\mathcal{A}$ rgs $\cup \mathcal{A r g s}^{+}$is maximal.

The following two theorems state that semi-stable semantics indeed coincides with reinstatement labellings in which undec is minimal.

Theorem 17. Let $(A r$, def $)$ be an argumentation framework and $\mathcal{L}$ be a reinstatement labelling such that undec $(\mathcal{L})$ is minimal. Then $\mathcal{A} r g s=\operatorname{Lab} 2 \operatorname{Ext}(\mathcal{L})$ is a semi-stable extension.

Proof. This follows directly from lemma 4 and definition 7 .

Theorem 18. Let (Ar, def) be an argumentation framework and $\mathcal{A}$ rgs be a semi-stable extension. Then $\mathcal{L}=\operatorname{Ext} 2 \operatorname{Lab}(\mathcal{A r g s})$ is a reinstatement labelling such that undec $(\mathcal{L})$ is minimal.

Proof. This follows directly from lemma 3 and definition 7 .

An interesting property of semi-stable extensions is the following.

Theorem 19. Let $(A r, d e f)$ be an argumentation framework. If there exists a stable extension, then the semi-stable extensions coincide with the stable extensions.

Proof. Suppose there exists a stable extension $\mathcal{A}$ rgs. Let $\mathcal{L}=\operatorname{Ext2Lab}(\mathcal{A} r g s)$. From Theorem 5 it follows that undec $(\mathcal{L})=\emptyset$. As a semi-stable extension minimizes undec (Theorem 18), the fact that $\mathcal{A}$ rgs has empty undec means that in the particular argumentation framework $(A r, d e f)$ every semi-stable extension should have empty undec. This means that in $(A r, d e f)$ every semi-stable extension is a stable extension. The fact that every stable extension is a semi-stable extension follows from the fact that the empty set is the minimal element w.r.t. set-inclusion.

It should be mentioned that theorem 19 does not hold when semi-stable semantics is replaced by preferred semantics. That is, it is not the case that if there exists a stable extension, the preferred extensions coincide with the stable extensions (see figure 2 for a counterexample). Semi-stable semantics is thus very close to stable semantics (closer than, for instance, preferred semantics) without the traditional disadvantage of stable semantics (the potential absence of extensions).

The idea of semi-stable semantics is not entirely new. It is quite similar to Verheij's concept of an admissible stage extension, which fits within Verheij's general approach of using stages to deal with the issue of argument reinstatement [17].

Definition 8. An admissible stage extension is a pair $\left(\mathcal{A r g s}\right.$, Args $\left.^{+}\right)$where $\mathcal{A r g s}$ is an admissible set of arguments and $\mathcal{A}$ rgs $\cup \mathcal{A}$ rgs ${ }^{+}$is maximal. 
Theorem 20. Let $($ Ar, def $)$ be an argumentation framework and $\mathcal{A r g s} \subseteq \operatorname{Ar}$. $\left(\mathcal{A r g s}, \operatorname{Args}^{+}\right)$ is an admissible stage extension iff $\mathcal{A}$ rgs is a semi-stable extension.

Proof. We basically have to prove the following (definitions 8 and 7):

$\mathcal{A}$ rgs is an admissible set where $\mathcal{A r g s} \cup \mathcal{A r g s}^{+}$is maximal iff

$\mathcal{A}$ rgs is a complete extension where $\mathcal{A r g s} \cup \mathcal{A r g s}^{+}$is maximal.

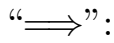

A complete extension is a stronger condition than an admissible set, so we only need to prove that an admissible set $\mathcal{A}$ rgs where $\mathcal{A r g s} \cup \mathcal{A r g s}^{+}$is maximal is also a complete extension. Suppose this is not the case. Then there must be an argument $B \notin \mathcal{A}$ rgs that is defended by $\mathcal{A}$ rgs. This means that every argument $C$ that defeats $B$ is defeated by an argument in Args. Therefore, $B \notin \mathcal{A r g s}^{+}$(otherwise $\mathcal{A r g s}$ would not be conflict-free). This means that Args $\cup\{B\}$ is conflict-free and self-defending, and thus an admissible set. But this would mean that $\mathcal{A}$ rgs is not an admissible set for which $\mathcal{A r g s} \cup \mathcal{A r g s}^{+}$is maximal. Contradiction. "£":

An admissible set is a weaker condition than a complete extension. We therefore only need to prove that maximality still holds under this weaker condition. Suppose that $\mathcal{A r g s} \cup \mathcal{A r g s}^{+}$ would not be maximal. This means there exists an admissible set $\mathcal{A}$ rgs ${ }^{\prime}$ such that $\left(\mathcal{A}\right.$ rgs ${ }^{\prime} \cup$

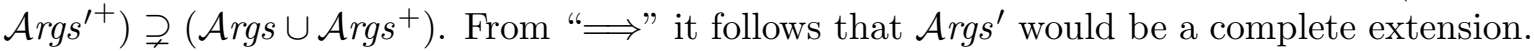
But then $\mathcal{A}$ rgs would not have been a complete extension where $\mathcal{A r g s} \cup \mathcal{A r g s}^{+}$is maximal. Contradiction.

\subsection{Overview}

From the previous discussion, it is clear that there exists a connection between the various forms of reinstatement labellings on one hand and the various Dung-style semantics on the other hand. This connection is summarized in table 1.

\begin{tabular}{|c|c|c|}
\hline $\begin{array}{c}\text { restriction } \\
\text { reinst. labellings }\end{array}$ & $\begin{array}{c}\text { Dung-style } \\
\text { semantics }\end{array}$ & $\begin{array}{c}\text { linked by } \\
\text { theorems }\end{array}$ \\
\hline \hline no restrictions & complete semantics & 1 and 2 \\
\hline empty undec & stable semantics & 4 and 5 \\
\hline maximal in & preferred semantics & 6 and 7 \\
\hline maximal out & preferred semantics & 8 and 9 \\
\hline maximal undec & grounded semantics & 10 and 11 \\
\hline minimal in & grounded semantics & 12 and 13 \\
\hline minimal out & grounded semantics & 14 and 15 \\
\hline minimal undec & semi-stable semantics & 17 and 18 \\
\hline
\end{tabular}

Table 1: Reinst. labellings versus Dung-style semantics.

There also exists a partial ordering between the various Dung-style semantics. Every stable extension is a semi-stable extension, every semi-stable extension is a preferred extension, every preferred extension is a complete extension, and every grounded extension is a complete extension. This is graphically depicted in figure 3 . 


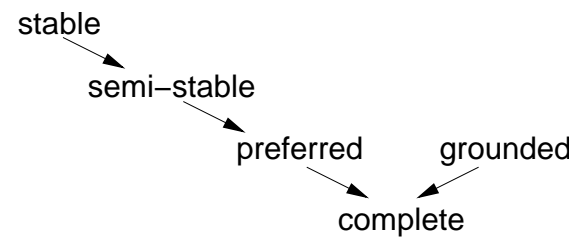

Figure 3: An overview of the different semantics.

\section{Semantics Revisited}

In essence, a reinstatement labelling can be seen as a subjective but reasonable point of view that an agent can take with respect to which arguments are in, out or undec. Each such position is internally coherent in the sense that, if questioned, the agent can use its own position to defend itself. It is possible for the position to be disagreed with, but at least one cannot point out an internal inconsistency. The set of all reinstatement labellings therefore stands for all possible and reasonable positions an agent can take.

When determining the overall justified arguments, two approaches are possible: the sceptical and the credulous one. Under the credulous approach, an argument is justified iff there is at least one reasonable position (= reinstatement labelling) where it is labelled in. Under the sceptical approach, an argument is justified iff it is in in every reasonable position; that is, a reasonable agent cannot deny that the argument is in. As reinstatement labellings coincide with complete extensions (as was explained in section 4.1), it would seem that credulous and sceptical inference could be modelled by applying complete semantics.

It is interesting to compare complete semantics with some current approaches. Let us consider the example of figure 4

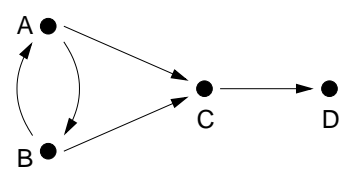

Figure 4: A floating argument.

In the case of the argumentation framework of figure 4 there are three reinstatement labellings, as stated in figure 5 .

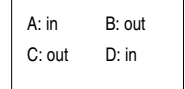

L1

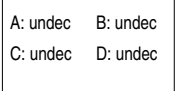

L2

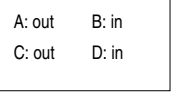

L3

Figure 5: Three reinstatement labellings.

When all reinstatement labellings are taken into account (such is the case in complete semantics) then $A, B$ and $D$ are credulously justified, whereas no arguments are sceptically justified. 
It is interesting to compare this approach with preferred semantics, which has been the subject of much recent research $[19,7,6]$. As was explained earlier, a preferred extension coincides with a reinstatement labelling in which the set of arguments labelled in is maximal. In case of figure 4 , for instance, the relevant labellings are only $\mathcal{L}_{1}$ and $\mathcal{L}_{3}$; thus, $\mathcal{L}_{2}$ is ruled out (see figure 6).

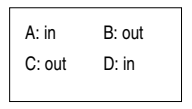

L1

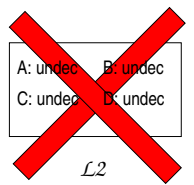

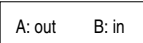

C: out D: in

L3

Figure 6: Preferred semantics rules out particular labellings.

What preferred semantics essentially does is to rule out zero or more reinstatement labellings before determining which arguments are credulously or sceptically justified. Under the sceptical approach, this can lead to more conclusions becoming justified. In the case of figure 4 , for instance, argument $D$ is sceptically justified under preferred semantics but not under complete semantics.

The fact that under preferred semantics, reinstatement labelling $\mathcal{L}_{2}$ is ruled out can be seen as odd. $\mathcal{L}_{2}$, after all, is a perfectly valid reinstatement labelling. The fact that it is ruled out under preferred semantics means that those who defend preferred semantics must have some reason to justify this. This reason should state why $\mathcal{L}_{2}$ is "wrong" or "irrelevant", thus making it possible to ignore $\mathcal{L}_{2}$. One such reason could be (theorems 6 and 7 ) " $\mathcal{L}_{2}$ should be ignored because the set of in-labelled arguments is not maximal." This reason does not appear to be a very strong one.

A more pragmatic reason in favor of preferred semantics is the issue of floating conclusions and floating arguments. Suppose the following information is available: (1) Lars's mother is Norwegian, (2) Lars's father is Dutch, (3) Norwegians like ice-skating and (4) Dutch like ice-skating. We can now construct two arguments that defeat each other: (A) Lars likes ice-skating because he's Norwegian and (B) Lars likes ice-skating because he's Dutch. Under sceptical complete semantics, the proposition that Lars likes ice-skating is not justified, despite the fact that, intuitively, it should be. Under sceptical preferred semantics, on the other hand, the proposition that Lars likes ice-skating is justified. At a first sight, this seems to illustrate a clear advantage of preferred semantics to complete semantics.

If we take a closer look, however, the situation becomes more complex. This is because the issue of whether or not Lars likes ice-skating depends on whether or not the principle of excluded middle is regarded as valid. In monotonic logic, the validity of a statement $p \vee \neg p$ depends on the number of truth-values. Whereas in a two-valued logic (where each proposition is either true or false in a given model) the proposition $p \vee \neg p$ is usually regarded as valid, it is not regarded as valid in, for instance, three-valued logics [16, 10]. Similarly, for one of the two conflicting arguments $A$ and $B$ to be regarded as valid (or justified), one should require that an argument is either in or out, resulting in a two-valued reinstatement labelling (without undec). In section 4.2, it was shown that this essentially boils down to stable semantics. Stable semantics, however, suffers from the problem that for some argumentation frameworks, no stable extensions exist. Consequently, it is not always possible to have a reinstatement labelling with only in and out. A third possibility (undec) 
is needed. Therefore, the principle of the excluded middle, as an absolute criterion, should be rejected. ${ }^{1}$ For those who nevertheless feel that the principle of the excluded middle should perhaps not hold at all times, but at least as much as possible (thus not completely ruling out undec but merely minimizing it), semi-stable semantics would seem a more appropriate choice than preferred semantics.

Given the observation that the principle of complete semantics can be given a decent philosophical justification, it is interesting to examine how complete semantics could be implemented. Fortunately, it turns out that both sceptical and credulous complete semantics have relatively easy and well-documented proof procedures.

As for sceptical semantics, an argument is in each complete extension iff it is in the grounded extension.

Theorem 21 ([8]). Let $\left\{C E_{1}, \ldots, C E_{n}\right\}$ be the set of complete extensions and $G E$ be the grounded extension. Let $A$ be an argument. It holds that $A \in G E$ iff $A \in C E_{1} \cap \ldots \cap C E_{n}$.

As for credulous semantics, an argument is in some complete extension iff it is in some admissible set.

Theorem 22. Let $C E_{1}, \ldots, C E_{n}$ be the set of complete extensions and $A S_{1}, \ldots, A S_{m}$ be the set of admissible sets. Let $A$ be an argument. It holds that $\exists C E_{i} \in\left\{C E_{1}, \ldots, C E_{n}\right\}: A \in C E_{i}$ iff $\exists A S_{j} \in\left\{A S_{1}, \ldots, A S_{m}\right\}: A \in A S_{j}$.

Proof.

"—":

Suppose $A$ is in some complete extension $C E_{i}$. AS $F\left(C E_{i}\right)=C E_{i}$, it holds that $\mathcal{A} r g s$ is admissible. Therefore, $A$ is in some admissible set.

"«":

Suppose $A$ is in some admissible set $A S_{j}$. Then there also exists a maximal admissible set $\mathcal{A}$ Args $^{\prime}$ such that $A S_{j} \subseteq \mathcal{A r g s}^{\prime}$. By definition, this maximally admissible set is a preferred extension. Furthermore, every preferred extension is also a complete extension [8]. This means that $A$ is also in some complete extension.

The fact that sceptical complete semantics coincides with grounded semantics, and credulous complete semantics coincides with credulous preferred semantics is advantageous, as these have relatively straightforward and well-studied proof procedures. Proof procedures for grounded semantics are given in [15, 2], and proof procedures for credulous preferred semantics are given in $[19,5]$.

\section{Summary and Discussion}

In this paper, we showed it is possible to describe Dung's standard semantics in terms of reinstatement labellings, which provide an intuitive and relatively simple way of dealing with the issue of reinstatement. We also showed how reinstatement labellings can be used to pinpoint the exact differences between Dung's standard semantics. Using a systematic analysis of reinstatement labellings, we were also able to specify an additional form of semantics (semi-stable

\footnotetext{
${ }^{1}$ Another issue where the principle of the excluded middle does not hold in most formalisms for defeasible reasoning is in handling disjunctive information. If $\{p \vee q\} \subseteq \mathcal{P}$ and $\{p \Rightarrow r ; q \Rightarrow r\} \subseteq \mathcal{D}$ then in most formalisms for defeasible reasoning, $r$ is not justified, although intuitively it should be, if one accepts the principle of the excluded middle.
} 
semantics) and showed how this semantics fits into the overall picture (figure 3). We then reexamined the various semantical approaches and made a case for grounded semantics for sceptical entailment and credulous preferred semantics for credulous entailment. ${ }^{2}$

One of the researchers who has done some work on the relation between reinstatement labellings ("status assignments") and Dung's various semantics is Prakken. In particular, Prakken proves (in his own terms and particular formalization) that reinstatement labellings without undec correspond to stable extensions, and that reinstatement labellings with maximal in correspond to preferred extensions [14]. It was the work of Prakken that served as an inspiration for the more thorough analysis in this paper.

Other recent work on reinstatement labellings has been done by Jakobovits and Vermeir [12]. Their definition of a labelling, however, is different than ours. First of all, they allow for an argument to be labelled in, out, both in and out, or neither in or out. Furthermore, their main reinstatement postulate is different.

Definition 9 ([12], syntax and formulation adjusted). $\mathcal{L}$ is a labelling iff:

- $\forall A \in A r:(\mathcal{L}(A)=$ out $\equiv \exists B \in A r:(B \operatorname{def} A \wedge \mathcal{L}(B)=$ in $))$ and

- $\forall A \in A r:(\mathcal{L}(A)=$ in $\supset \forall B \in A r:(B \operatorname{def} A \supset \mathcal{L}(B)=$ out $))$.

The difference between definition 9 and the earlier presented definition 5 is that the former does not require an argument of which all defeaters are out to be labelled in. This is quite strange, since it also means that an argument that has no defeaters at all is not required to be labelled in. To some extent, this problem is repaired for complete labellings, in which each argument is labelled either in, out or both.

The overall aim of Jakobovits and Vermeir is to come up with a semantics that is different from Dung's. Jakobovits and Vermeir justify their approach by discussing a number of small examples. However, the general approach of using examples in order to justify a particular formalism has some important downsides. To illustrate our main point, consider the following example provided in [12].

\section{Example 2.}

A: If the bacteria in the patient's blood is not of type $X$ then it must be of type $Y$.

$B$ : If the bacteria in the patient's blood is not of type $Y$ then it must be of type $X$.

$C$ : If the patient does not have bacterial infection then giving antibiotics to the patient is superfluous.

D: If it is not superfluous to give the patient antibiotics then the antibiotics should be prescribed.

Example 2 is represented in the argumentation framework of figure 7.

Jakobovits and Vermeir argue that the correct outcome should be that argument $D$ is justified. However, it is quite easy to provide another example, with essentially the same structure, where the desired outcome is totally different.

\footnotetext{
${ }^{2}$ This also implies that we do not support the approach of sceptical preferred semantics, as is for instance examined by [7]. We reject sceptical preferred semantics for reasons discussed in the previous section. We do, however, support the approach of credulous preferred semantics, as this coincides with credulous complete semantics.
} 


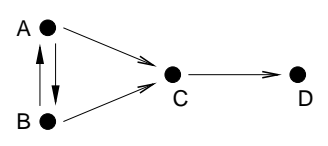

Figure 7: The argumentation framework of example 2 and 3.

\section{Example 3.}

A: The suspect killed the victim by stabbing him with a knife.

B: The suspect killed the victim by shooting him with a gun.

$C$ : The suspect is innocent.

D: The suspect should go to jail.

This essentially gives the same argumentation framework as figure 7. However, an analysis of this case yields a different outcome. As essentially none of the witness statements is without doubt, none of them can serve as a good reason to refute the innocence of the suspect, and the conclusion that suspect should go to jail is definitely not an intuitive or desired one.

The main point here is that some researchers try to justify a particular design decision by giving an abstract example (like figure 7) an informal meaning (like example 2 or example 3 ) and then arguing that the outcome of the abstract example should be in line with the "intuitive" outcome of the informal example. Although this approach has been applied by various researchers in the past, it has also been criticized $[18,2]$ for its inherent ad-hoc nature.

It is the author's opinion that a better justification for the design of a particular logical formalism can be found in postulates, as these have a more general nature than separate examples. And for reasons explained earlier, we feel that definition 5 can serve as a more intuitive and acceptable postulate for reinstatement than definition 9. It is the author's firm opinion that Dung's traditional semantics have a solid basis and that one should have very good reasons for adjusting them.

\section{References}

[1] ASPIC-consortium. Deliverable D2.5: Draft formal semantics for ASPIC system, June 2005.

[2] M. Caminada. For the sake of the Argument. Explorations into argument-based reasoning. Doctoral dissertation Free University Amsterdam, 2004.

[3] M. Caminada. Collapse in formal argumentation systems. Technical Report UU-CS2005-023, Utrecht University, 2005.

[4] M. Caminada and L. Amgoud. An axiomatic account of formal argumentation. In Proceedings of the AAAI-2005, pages 608-613, 2005.

[5] C. Cayrol, S. Doutre, and J. Mengin. Dialectical Proof Theories for the Credulous Preferred Semantics of Argumentation Frameworks. In Proceedings of the 6th European Conference on Symbolic and Quantitative Approaches to Reasoning with Uncertainty (ECSQARU-2001), volume 2143 of LNAI, pages 668-679. Springer-Verlag, 2001. 
[6] Y. Dimopoulos, B. Nebel, and F. Toni. Finding Admissible and Preferred Arguments Can be Very Hard. In Proc. of the 7th Int. Conf. on Principles of Knowledge Representation and Reasoning (KR-2000), pages 53-61, 2000.

[7] S. Doutre and J. Mengin. On sceptical versus credulous acceptance for abstract argument systems. In Proceedings of the 9th European Conference on Logics in Artificial Intelligence (JELIA-2004), pages 462-473, 2004.

[8] P. M. Dung. On the acceptability of arguments and its fundamental role in nonmonotonic reasoning, logic programming and $n$-person games. Artificial Intelligence, 77:321-357, 1995.

[9] G. Governatori, M.J. Maher, G. Antoniou, and D. Billington. Argumentation semantics for defeasible logic. Journal of Logic and Computation, 14(5):675-702, 2004.

[10] R. Hähnle. Advanced many-valued logic. In D. Gabbay and F. Günthner, editors, Handbook of Philosophical Logic, volume 2, pages 297-395. Kluwer Academic Publishers, Dordrecht/Boston/London, second edition, 2001.

[11] J. Horty. Argument construction and reinstatement in logics for defeasible reasoning. Artificial Intelligence and Law, 9:1-28, 2001.

[12] H. Jakobovits and D. Vermeir. Robust semantics for argumentation frameworks. Journal of logic and computation, 9(2):215-261, 1999.

[13] H. Prakken. Intuitions and the modelling of defeasible reasoning: some case studies. In Proceedings of the Ninth International Workshop on Nonmonotonic Reasoning (NMR2002), pages 91-99, Toulouse, France, 2002.

[14] H. Prakken. Commonsense reasoning. Technical report, Institute of Information and Computing Sciences, Utrecht University, 2004. Reader.

[15] H. Prakken and G. Sartor. Argument-based extended logic programming with defeasible priorities. Journal of Applied Non-Classical Logics, 7:25-75, 1997.

[16] A. Urquhart. Basic many-valued logic. In D. Gabbay and F. Günthner, editors, Handbook of Philosophical Logic, volume 2, pages 249-295. Kluwer Academic Publishers, Dordrecht/Boston/London, second edition, 2001.

[17] B. Verheij. An integrated view on rules and principles. In R.W. van Kralingen et al., editor, Legal Knowledge Based Systems. Foundations of Legal Knowledge Systems (Proceedings of JURIX-1996), pages 25-38, Tilburg, 1996. Tilburg University Press.

[18] G. A. W. Vreeswijk. Studies in defeasible argumentation. PhD thesis at Free University of Amsterdam, 1993.

[19] G. A. W. Vreeswijk and H. Prakken. Credulous and sceptical argument games for preferred semantics. In Proceedings of the 7th European Workshop on Logic for Artificial Intelligence (JELIA-00), number 1919 in Springer Lecture Notes in AI, pages 239-253, Berlin, 2000. Springer Verlag. 